\section{OPEN ACCESS}

Edited by:

Marzia Di Donato

University of Campania Luigi Vanvitelli,

Italy

Reviewed by:

Aria Baniahmad,

University Hospital Jena, Germany

Hiroshi Miyamoto,

University of Rochester Medical

Center, United States

Costanza Maria Cristiani,

Magna Græcia University

of Catanzaro, Italy

${ }^{*}$ Correspondence:

Jan Geliebter

jan_geliebter@nymc.edu

tPresent address:

Timmy J. O'Connell,

Department of Bioinformatics $R \& D$,

Sema4, Stamford, CT, United States

¥These authors share first authorship

Specialty section:

This article was submitted to Molecular and Cellular Oncology, a section of the journa Frontiers in Cell and Developmental Biology

Received: 02 February 2021 Accepted: 23 June 2021

Published: 06 August 2021

Citation:

O'Connell TJ, Dadafarin S, Jones $M$, Rodríguez T, Gupta $A$,

Shin E, Moscatello A, lacob C, Islam H, Tiwari RK and Geliebter J

(2021) Androgen Activity Is Associated With PD-L1

Downregulation in Thyroid Cancer. Front. Cell Dev. Biol. 9:663130. doi: 10.3389/fcell.2021.663130

\title{
Androgen Activity Is Associated With PD-L1 Downregulation in Thyroid Cancer
}

\section{Timmy J. O’Connell'1+‡, Sina Dadafarin ${ }^{1 \neq}$, Melanie Jones², Tomás Rodríguez ${ }^{3,4}$, Anvita Gupta 1 , Edward Shin 5 , Augustine Moscatello6, Codrin lacob7 , Humayun Islam', Raj K.Tiwari ${ }^{1}$ and Jan Geliebter ${ }^{1,6 *}$}

${ }^{1}$ Department of Pathology, Microbiology and Immunology, New York Medical College, Valhalla, NY, United States, ${ }^{2}$ United States Military Academy Preparatory School, West Point, NY, United States, ${ }^{3}$ RNA Therapeutics Institute, University of Massachusetts Medical School, Worcester, MA, United States, ${ }^{4}$ Medical Scientist Training Program, University of Massachusetts Medical School, Worcester, MA, United States, ${ }^{5}$ Department of Otolaryngology, New York Eye and Ear Infirmary, New York, NY, United States, ${ }^{6}$ Department of Otolaryngology, New York Medical College, Valhalla, NY, United States, ${ }^{7}$ Department of Pathology, New York Eye and Ear Infirmary, New York, NY, United States

Thyroid cancer is the most prevalent endocrine malignancy in the United States with greater than 53,000 new cases in 2020. There is a significant gender disparity in disease incidence as well, with women developing thyroid cancer three times more often than men; however, the underlying cause of this disparity is poorly understood. Using RNA-sequencing, we profiled the immune landscape of papillary thyroid cancer (PTC) and identified a significant inverse correlation between androgen receptor (AR) levels and the immune checkpoint molecule PD-L1. The expression of PD-L1 was then measured in an androgen responsive-thyroid cancer cell line. Dihydrotestosterone (DHT) treatment resulted in significant reduction in surface PD-L1 expression in a time and dose-dependent manner. To determine if androgen-mediated PD-L1 downregulation was AR-dependent, we treated cells with flutamide, a selective AR antagonist, and prior to DHT treatment to pharmacologically inhibit AR-induced signaling. This resulted in a $>90 \%$ restoration of cell surface PD-L1 expression, suggesting a potential role for AR activity in PD-L1 regulation. Investigation into the AR binding sites showed AR activation impacts NF-kB signaling by increasing $\mathrm{IkB} \alpha$ and by possibly preventing NF-kB translocation into the nucleus, reducing PD-L1 promoter activation. This study provides evidence of sex-hormone mediated regulation of immune checkpoint molecules in vitro with potential ramification for immunotherapies.

Keywords: PD-L1 pathway, androgen receptor, immune surveillance, gender disparity, thyroid cancer

\section{INTRODUCTION}

Thyroid cancer is the most common endocrine cancer and accounts for 3.8\% of all cancers (Nguyen et al., 2015). According to the Surveillance, Epidemiology, and End Results Program (SEER), the incidence of thyroid cancer has increased by threefold over the past three decades (Kilfoy et al., 2009) though stabilized during this past decade (Morris et al., 2016). Of the 53,000 new thyroid cancer cases that occurred in 2020, approximately 40,000 occurred in women and 13,000 occurred 
in men (Siegel et al., 2020). Age-adjusted SEER incidence rates show a three to fourfold increase in incidence of thyroid cancer in women aged 20-49 as compared to men aged 20-49. A study of 566 papillary thyroid cancer (PTC) patients similarly concluded that women have a higher incidence of PTC than males, and also found that men tended to present with larger carcinomas (Yorke et al., 2016). Previously, it was shown that female gender is associated with a high prevalence of RET/PTC1 and RET/PTC3 fusions in PTC (Su et al., 2016). Changes in estrogen receptor (ER) and androgen receptor (AR) have been observed in PTC, with increases in $\mathrm{ER} \alpha$, decreases in $\mathrm{ER} \beta$, and decreases in AR being associated with a more aggressive phenotype (Magri et al., 2012). Rajoria et al. (2010) showed that a metastatic thyroid cancer phenotype is regulated by estrogen and functional ER which enhance mitogenic, migratory, and invasive properties of thyroid cancer cells. ER and AR expression changes may be acting as a cause or a result of the onset of PTC (Magri et al., 2012). Additionally, it was previously suggested that underlying inflammatory processes may predispose healthy females toward developing thyroid cancer (Manole et al., 2001; Tafani et al., 2014).

The gender disparity in thyroid cancer incidence is a unique phenomenon, whereas nearly all other cancers without obvious anatomical bias are neutral or have a bias toward higher incidence in men. Additionally, males and females differ in their immunological responses and show unique innate and adaptive immune responses (Klein and Flanagan, 2016). It has been shown that females in general have a more active humoral and cellular immune response than men (Ansar Ahmed et al., 1985). Women are more prone to autoimmune and inflammatory thyroid diseases such as Graves' Disease or Hashimoto's Thyroiditis (Ngo et al., 2014). Therefore, underlying inflammatory processes in healthy females may predispose them toward developing thyroid cancer (Manole et al., 2001; Tafani et al., 2014). Despite the well-established characterization of sex disparity in thyroid cancer incidence and that inflammation plays a key role in the gender disparity of some cancers (Naugler et al., 2007), the underlying molecular factors that mediate this difference are poorly understood (Rahbari et al., 2010).

Recently, evidence has emerged showing sex differences in the expression of immune checkpoint molecules on tumors (Özdemir and Dotto, 2019; Wilms et al., 2020) and the response to checkpoint inhibition (Nosrati et al., 2017). A comprehensive analysis of the immune landscape of cancer across The Cancer Genome Atlas (TCGA) dataset specifically found the Programmed Death Receptor Ligand 1 (PD-L1) was more highly expressed among women than in men in thyroid carcinoma and three other cancer types (head and neck squamous cell, renal cell carcinoma, and lung adenocarcinoma) (Thorsson et al., 2019). Furthermore, PD-L1 has consistently been shown to be highly expressed in the most aggressive forms of thyroid cancer (Ahn et al., 2017; Chintakuntlawar et al., 2017).

Given the role gender may play in the incidence of thyroid cancer, we hypothesized that sex hormones may modulate the expression of immune checkpoint molecules, and explaining the sex disparity in their expression. In this study, we present data that suggest that $\mathrm{AR}$ activation attenuates $\mathrm{PD}-\mathrm{L} 1$ expression in a thyroid cancer cell line, potentially by inhibiting NF-kB signaling by increasing the $\mathrm{IkB} \alpha$ inhibitory subunit. Our study associates the role of androgens in the gender-specific expression patterns of PD-L1 and suggests the possibility of targeting sex hormone pathways in tandem with immunotherapeutics.

\section{MATERIALS AND METHODS}

\section{Patient Specimens}

This study was approved by the institutional review boards of New York Eye and Ear Infirmary and New York Medical College (NYMC). Written informed consent was obtained from each patient. All TCGA data used in this study were downloaded from publicly available sources. Specimens were obtained from 44 patients undergoing thyroidectomy and fresh frozen thyroid tissue were collected between 2009 and 2013. All tumors had corresponding matched normal adjacent tissue and diagnosis of PTC was validated by pathological examination.

\section{RNA Sequencing}

Total RNA was extracted from frozen specimens using TRIzol Reagent (Thermo Fisher Scientific, Waltham, MA, United States) and RNA cleanup was performed using RNeasy Plus Mini Kit (Qiagen, Hilden, Germany) according to manufacturer's instructions. RNA quality, concentration, and fragment size distribution were assessed on a 2100 Bioanalyer (Agilent Inc., Palo Alto, CA, United States). Ribosomal RNA was depleted using the Illumina Ribo-Zero rRNA removal kit. Samples were aligned to the human genome using STAR software version 2.4 (Dobin et al., 2013) and quantified using RSEM version 1.2.14 (Li and Dewey, 2011). KEGG Pathway and gene ontology (GO) enrichment analyses were performed on Advaita's iPathwayGuide $^{1}$ Platform using DE genes $[\operatorname{abs}(\log 2 \mathrm{FC})>1.5$ and $q$-value $<0.05]$. Statistical tests of pathway and GO term enrichment were adjusted using FDR correction.

\section{TCGA Data}

Level three RNA-Sequencing data from TCGA project were downloaded from the UCSC Xena Browser (Goldman et al., 2018).

\section{Cell Culture}

The 8505C cell line was purchased from MilliporeSigma (ECACC Cat\# 94090184, RRID:CVCL_1054) and the K1 cell line was graciously provided as a gift from the Schweppe lab. 84e7, K1-lentiAR, and 8505C-lentiAR cell lines were maintained in phenol-red free RPMI 1640 supplemented with $10 \mathrm{mM}$ L-Glutamine, $100 \mathrm{U} / \mathrm{mL}$ penicillin/streptomycin (Corning, 30002CI, Manassas, VA, United States) and 5\% United States origin, heat-inactivated fetal bovine serum (FBS) (Genesee Scientific, 25-514 H, El Cajon, CA, United States). $84 \mathrm{e} 7$ cells also were supplemented with $50 \mu \mathrm{g} / \mathrm{mL}$ G418 sulfate solution (Corning, 30-234-CR, Manassas, VA). Thyroid cancer

\footnotetext{
${ }^{1}$ http://www.advaitabio.com/ipathwayguide
} 
cell line gene expression data was obtained from the Cancer Cell Line Encyclopedia (CCLE) ${ }^{2}$.

\section{Lentiviral Transduction}

HEK293T cells were purchased from ATCC (ATCC Cat\# CRL3216, RRID:CVCL_0063) and cultured in DMEM supplemented with $10 \mathrm{mM}$ L-Glutamine, $100 \mathrm{U} / \mathrm{mL}$ penicillin/streptomycin and $10 \%$ heat inactivated FBS. The pLENTI6.3/AR-GC-E2325 plasmid (kindly shared by the Kalland lab, Addgene plasmid \# 8512833; RRID:Addgene_85128) along with 0.4 ug of psPAX2 packaging and 0.15 ug of VSV-G envelope plasmids were transfected into HEK293T cells using the TransIT-LT1 reagent (Mirus, MIR 2304, Madison, WI, United States). Viruscontaining supernatants were collected at $48 \mathrm{~h}$ post-transfection and filtered using a 0.45 um PES filter. $8505 \mathrm{C}$ and $\mathrm{K} 1$ cells were treated with $40 \mathrm{ng} / \mathrm{mL}$ polybrene (MilliporeSigma \#TR-1003-G, St. Louis, MO, United States) and filtered virus for $24 \mathrm{~h}$ followed by replacement with $10 \mathrm{ug} / \mathrm{mL}$ Blasticidin containing media. After $72 \mathrm{~h}$ of Blasticidin selection, cells were then sub-cultured and frozen for future experiments.

\section{Western Blotting}

Cultured cells were lysed in RIPA Buffer $(50 \mathrm{mM} \mathrm{Tris-} \mathrm{Hcl} \mathrm{pH}$ $7.4,150 \mathrm{mM} \mathrm{NaCl}, 0.25 \%$ sodium deoxcholate, and 1.0\% NP-40) supplemented with HALT Protease Inhibitor Cocktail (Thermo Fisher Scientific/ Pierce, P/N 78430). 15 ug of total protein from each cell extract were added to $5 \mathrm{uL}$ of sample buffer (10\% $\beta$-mercaptoethanol in $4 \times$ Laemmli buffer) followed by SDS-PAGE electrophoresis performed under reducing conditions with a $4 \%$ stacking gel and $10 \%$ separating gel. Proteins were transferred from the gel to an Immobilon-P PVDF Membrane (MilliporeSigma, P/N IPVH00010). Membranes were incubated with primary antibodies diluted in 5\% milk (total protein) or $1 \%$ milk (phosphorylated protein) in $1 \times$ TBST overnight at $4^{\circ} \mathrm{C}$. Secondary goat anti-rabbit Horseradish Peroxidase (HRP) conjugated secondary antibody (Abcam Cat\# ab6721, RRID:AB_955447) was diluted 1:5,000 in 5\% milk with $1 \times$ TBST and incubated with membrane for $2 \mathrm{~h}$ at room temperature. Quantitative analysis of optical density was performed with ImageJ. Antibodies used for western blotting can be found in Supplementary Table 1.

\section{Flow Cytometry}

A $0.5-1 \times 10^{6}$ cultured cells were collected using cell scrapers and resuspended in $100 \mu \mathrm{L}$ incubation buffer $(0.25 \mathrm{~g}$ bovine serum albumin dissolved in $50 \mathrm{~mL} 1 \times$ PBS) with $20 \mu \mathrm{L}$ of FITC-conjugated mouse anti-human primary PD-L1 monoclonal antibody (BD Pharmingen, $\mathrm{P} / \mathrm{N}$ 558065) for $1 \mathrm{~h}$ at room temperature. Cells were rinsed $2 \times$ in incubation buffer and finally resuspended in $0.45 \mathrm{~mL}$ ice cold $1 \times$ PBS and strained into polystyrene flow cytometry tubes. Flow Cytometry was conducted using the BD FACScan Flow Cytometer and each experiment was conducted in triplicate. Examples for gating parameters can be found in Supplementary Figure 1. Relative

${ }^{2}$ http://www.broadinstitute.org/ccle/home

${ }^{3}$ http://n2t.net/addgene:85128 fluorescence intensity (RFI) was calculated by normalizing mean fluorescence intensity (MFI) measurements of treated samples to vehicle control:

$$
R F I=\frac{M F I_{\text {treatment }}}{M F I_{\text {control }}}
$$

\section{Cleavage Under Targets and Tagmentation (CUT\&Tag) and Sequencing}

For CUT\&Tag, $10^{6}$ 8505C-AR cells treated with either DHT or vehicle control for $48 \mathrm{~h}$ were used. CUT\&Tag DNA were prepared according to Kaya-Okur et al. (2019). The AR primary Rabbit mAb (Cell Signaling \#5153) and Guinea Pig anti-Rabbit secondary antibody (Novus Biologicals \#NBP172763, Centennial, CO, United States) were used as well as the CUTANA ${ }^{\mathrm{TM}}$ pAG-Tn5 (Epicypher \#15-1017, Durham, NC, United States).

Samples were multiplexed and sequenced on a Nextseq 500 in paired-end mode (38/37) with a high-output, 75-cycle reagent kit. 3-prime read ends containing Nextera Mate Pair adapters were trimmed using cutadapt (Martin, 2011), filtering reads $<20 \mathrm{bp}$ $(-\mathrm{m} 20)$ or with an average phred quality score $<20$ (-q 20). Trimmed FASTQ files were aligned to the hg38 genome assembly using BWA mem (Li, 2013), marking duplicate reads (-M). Aligned SAM files were compressed to BAM format, sorted, merged, and indexed using samtools (Li et al., 2009). Duplicate reads were removed from BAM files using Picard Toolkit MarkDuplicates (Broad Institute, 2019).

To compare AR binding of genomic loci across biological conditions, we first used deeptools bamCoverage (Ramírez et al., 2014) to generate normalized BigWig files from deduplicated BAMs. Parameters selected include counts-permillion normalization (-normalizeUsing CPM) with a bin size of 10 bp $(-b s=10)$. Next, we calculated the aggregate normalized accessibility signal at known murine adult cis-regulatory elements (CREs) using UCSC bigWigAverageOverBed; these elements are subclassified as promoters, proximal enhancers and distal enhancers, and assigned a unique accession by ENCODE (ENCODE Project Consortium Moore et al., 2020). We used a Z-scoring method to identify high-confidence AR peaks in promoters. Total signal across cis-regulatory elements was recorded for each replicate using bigWigAverageOverBed. Only elements ranked in the top 10 percent $(Z>1.64)$ by signal were retained. The intersect of each replicate's top elements constitutes our high-confidence element list.

\section{Statistical Analysis}

Data analysis was performed on Prism version 8.1.2 (GraphPad Software, San Diego, CA, United States) and R version 3.6.2. Unless otherwise indicated, all experimental data were expressed as the mean \pm SD of experiments performed in triplicate. For analysis of paired NYMC tumor and normal tissue, a two-tailed, paired Student's $t$-test was performed. For comparison of tumor and normal TCGA data, a two-tailed Welch's $t$-test was used for statistical analysis. For all culture experiments, statistical significance of results was analyzed by two-tailed, and paired 
Student $t$-tests. For immunohistochemistry (IHC), Fisher's exact test was performed.

\section{RESULTS}

\section{Inflammation and AR Expression in Male and Female PTC}

To assess the difference in inflammatory and immune responses between male and female thyroid cancer, we performed RNAsequencing on 44 PTC with matched-paired normal thyroid tissue in the NYMC biobank ( 33 female and 11 male). A total of 1532 protein-coding genes and 756 non-coding RNAs underwent $\geq 1.5$-fold change between tumor and matched normal tissue ( $q$-value $\leq 0.05)$. Principal component analysis (PCA) on all specimens demonstrated distinct separation on the PC2 axis between PTC and normal adjacent tissue, indicating that $10 \%$ of transcriptomic variance was sufficient to distinguish tumor samples from normal tissue (Supplementary Figure 2A). KEGG pathway enrichment analysis of differentially expressed genes (DEGs) identified cell adhesion molecules and ECM-receptor interaction as well as pathways related to host immune function (Cytokine-cytokine receptor interactions and allograft rejection) (Supplementary Figure 2B). These findings are consistent with previous transcriptomic studies of PTC (Song et al., 2018).

Next, we examined GO terms enriched in DEGs from male and female PTC. A subset of GO biological processes related to adaptive immunity and inflammation were significantly enriched among female PTC but not male PTC (Figure 1A). Furthermore, genes that mediate T-cell activation such as CD40L, ICOS, and IL-2 were downregulated in female PTC (Supplementary Figure 3A).

Given the distinct gene expression patterns among immunerelated genes between female and male thyroid tissue, we next examined the expression of $\mathrm{AR}$ and its relationship to these gene expression patterns. AR expression in both the NYMC RNA-seq dataset and TCGA Thyroid Carcinoma project's RNAsequencing dataset showed significant $(p<0.0001)$ reduction in AR expression in tumors compared to normal tissue (Figure 1B). We performed a co-expression analysis between $\mathrm{AR}$ and a set of immune regulatory molecules, including CD28, CD80, CD86, CTLA-4, PD-1, PD-L2, TIM-3, TIM-3L, 4-1BB, 4-1BBL, OX40, and OX40L, in the NYMC dataset and found the expression of the immune checkpoint molecule CD274 had a significant inverse correlation (Pearson rho $=-0.60$ ) with AR and this finding was replicated with TCGA data (Figure 1C). Furthermore, males have a higher rate (70\%) of exhibiting an inverse relationship between AR and PD-L1 than females (35\%) $(p<0.05$, Supplementary Figure 3B).

\section{Androgen Downregulates PD-L1 in an AR-Dependent Mechanism}

Given the correlation between PD-L1 and AR expression in primary PTC tissue, we designed a series of cell culture experiments to test whether androgens regulate the expression of PD-L1 in thyroid cancer. The undifferentiated thyroid cancer cell line, $8505 \mathrm{C}$, was used due to the high surface level expression of PD-L1 (Cantara et al., 2019) and higher AR expression relative to other thyroid cancer cell lines based on mRNAsequencing and protein array data in the CCLE (Supplementary Figure 4). However, androgen treatment of the $8505 \mathrm{C}$ cell line failed to increase expression of the AR-responsive gene FKBP5 (Supplementary Figure 6A). We therefore used a transfected clone, termed 84e7 (Jones et al., 2021), that constitutively expresses human $\mathrm{AR}$ in a pcDNA3.1 vector under a $\mathrm{CMV}$ promoter. We initially measured PD-L1 levels by Western blot and detected an approximately $30 \%$ reduction $(p<0.01)$ in PD-L1 expression after $72 \mathrm{~h}$ of treatment with the potent androgen DHT but no change at $24 \mathrm{~h}$ (Supplementary Figure 5). Next, we quantified surface expression of PD-L1 using flow cytometry in $8505 \mathrm{C}$ and $84 \mathrm{e} 7$ cells treated with DHT or vehicle control at the $72 \mathrm{~h}$ timepoint. Cell surface PD-L1 levels decreased in $10 \mathrm{nM}$ DHT-treated $84 \mathrm{e} 7$ by approximately $60 \%$ $(p<0.005)$ as compared to vehicle-treated $84 \mathrm{e} 7$ while no change was observed between vehicle- and DHT-treated 8505C (Figure 2A). Notably, the DHT-mediated downregulation of PD-L1 was replicated in another thyroid cancer cell line, K1, and stably expressing the pLENTI6.3/AR-GC-E2325 construct (Supplementary Figure 6B). A dose response curve that encompassed all human physiological ranges of total androgens was used, from $0.1 \mathrm{nM}$ which is five times below the low end of the female physiological range, to $100 \mathrm{nM}$ which is three times above the high end of the male physiological range (Salameh et al., 2010; Figure 2B). Interestingly, the greatest impact of DHT occurred in the male physiological range, with a $50 \%$ reduction in $\mathrm{PD}$ L1 expression as compared to the female dose range. Next, we performed a time course of $10 \mathrm{nM}$ DHT treatment in $84 \mathrm{e} 7$ cells and found that the $60 \%$ reduction in PD-L1 surface expression was achieved by $48 \mathrm{~h}$ of treatment (Figure 2C).

To confirm that the cell surface decrease in PD-L1 observed in DHT-treated $84 \mathrm{e} 7$ was specific to AR signaling, $8505 \mathrm{C}$, and $84 \mathrm{e} 7$ were treated with varying concentrations of flutamide, a selective AR antagonist (Goldspiel and Kohler, 1990), prior to DHT treatment to pharmacologically inhibit AR-induced signaling. Flutamide was shown to inhibit the ability of DHT to decrease PD-L1 in $84 \mathrm{e} 7$, resulting in a dose-dependent and $>90 \%$ restoration of cell surface PD-L1 expression at $50 \mathrm{uM}$ concentration (Figure 2D), but not in 8505C cells (Supplementary Figure 6C) indicating that AR activity is necessary for DHT-mediated PD-L1 downregulation.

\section{CUT\&Tag Identifies AR Binding to Regulators of PD-L1}

To begin identifying potential mechanisms by which androgen signaling downregulates PD-L1 expression, we profiled AR binding genome wide using cleavage under targeted nuclease and tagmentation (CUT\&Tag) (Kaya-Okur et al., 2019). Since the $84 \mathrm{e} 7$ cell line required growth media supplemented with the G418 antibiotic that could confound results of genome-wide sequencing experiments, we generated an $8505 \mathrm{C}$ cell line stably expressing $\mathrm{AR}$ without requiring constant antibiotic selection via 


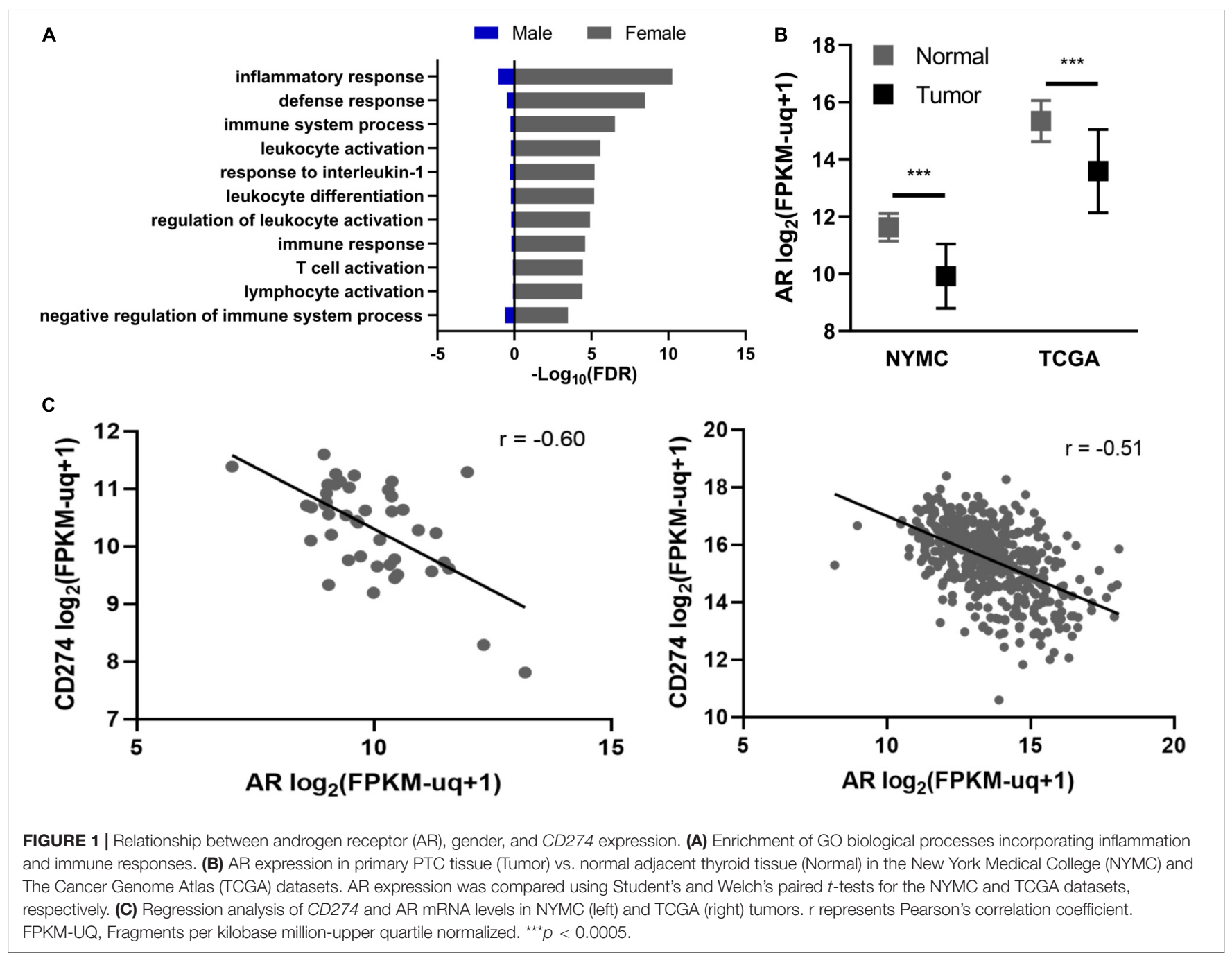

lentiviral transduction (Ryu et al., 2017). CUT\&Tag sequencing of this cell line (named $8505 \mathrm{C}$-lentiAR) after treatment with $10 \mathrm{nM}$ DHT for $48 \mathrm{~h}$ revealed direct AR binding to the PD-L1 gene body (Figure 3A), as well as potential regulatory elements downstream of the $\mathrm{PD}-\mathrm{L} 1$ open reading frame.

$\mathrm{PD}-\mathrm{L} 1$ expression is regulated by various signaling pathways including NFKB, MAPK, PI3K, mTOR, and JAK/STAT (Ritprajak and Azuma, 2015). We therefore surveyed our AR binding sites for promoter binding to molecules involved in these pathways and identified an AR binding signal at the NFKBIA promoter (Figure 3B). The NFKBIA gene encodes the $\mathrm{IkB} \alpha$ protein that sequesters NFkB in the cytoplasm, reducing NF-kB translocation to the nucleus. We confirmed that DHT increased both total and cytoplasmic IkB $\alpha$ proteins levels via western blot (Figure 3C and Supplementary Figure 7).

We next aimed to determine if androgen-mediated $\operatorname{IkB} \alpha$ upregulation contributes to the downregulation of PD-L1. 84e7 cells were treated with a selective and potent inhibitor of IKK, BMS-345541. Inhibition of IKK maintains $\mathrm{IkB} \alpha$ in the unphosphorylated state, resulting in sequestration of NF- $\kappa B$ in the cytoplasm (Burke et al., 2003). PD-L1 levels in 84e7 treated with BMS-345541 decreased significantly, but did not decrease PD-L1 surface expression to the same extent as $1 \mathrm{nM}$ or $10 \mathrm{nM}$ DHT (Figure 3D). When combined with DHT, there was no additive or synergistic effect on PD-L1 levels (Figure 3D). Thus, inhibition of IKK does indeed impact PD-L1 expression but does not account entirely for the decrease in PD-L1 observed with DHT, supporting a model where PD-L1 expression is modulated via $A R$ activity at multiple regulatory elements.

\section{DISCUSSION}

Despite the fact that thyroid cancer has a relatively quiet mutagenic landscape (Lawrence et al., 2013), there is increasing evidence that immune checkpoint molecules, specifically PDL1, and play a role in thyroid cancer pathogenesis (Chowdhury et al., 2016; Chintakuntlawar et al., 2017). Inflamed thyroid follicular cells express PD-L1 (Álvarez-Sierra et al., 2019) and immune checkpoint induced thyrotoxicity is a common adverse effect of PD1/PD-L1 blockade (D'Andréa et al., 2021). Furthermore, PD-L1 inhibitor-induced thyroiditis has been 


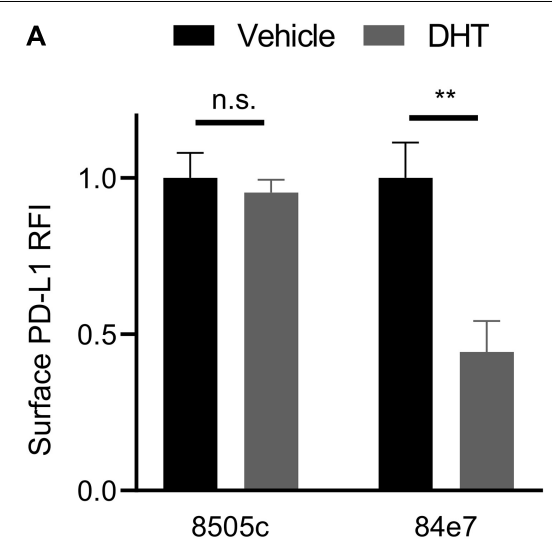

C

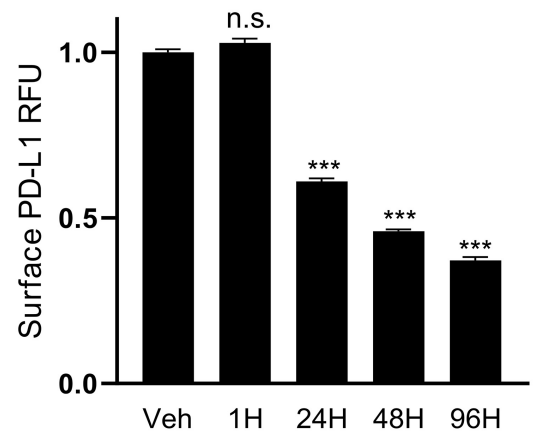

B

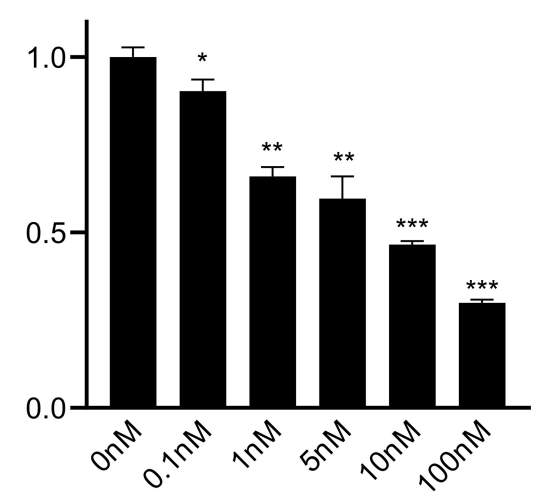

D

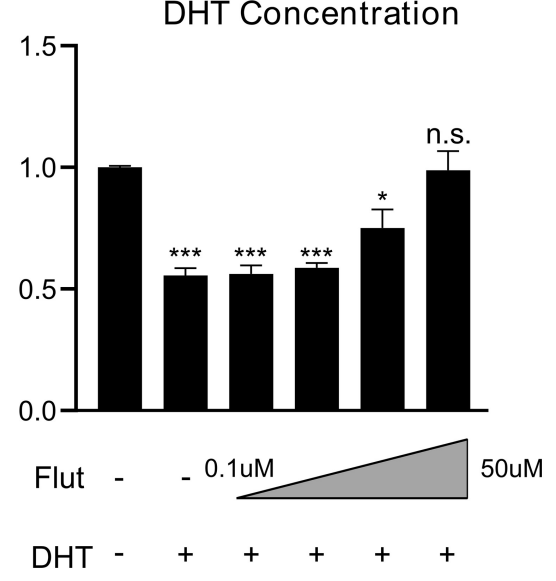

FIGURE 2 |PD-L1 is downregulated by androgen in an AR-dependent manner. (A) Surface expression of PD-L1 determined by flow cytometry in AR-lacking (8505C) or AR-expressing (84E7) thyroid cancer cell line after treatment with Vehicle (ethanol) or Dihydrotestosterone (DHT) (10 nM) for $72 \mathrm{~h}$. (B) Dose-response to variable DHT concentrations in $84 \mathrm{E} 7$ cells at $72 \mathrm{~h}$. (C) Time course of variable treatment lengths with 10 nM DHT. (D) Surface PD-L1 expression in $84 \mathrm{E} 7$ cell lines treated with 10 uM DHT and increasing concentrations of flutamide (Flut) at concentrations of 0.1, 1, 10, and 50 uM. RFI, relative fluorescence intensity. Student $t$-tests paired to control conditions ${ }^{\star} p<0.05 ;{ }^{* \star} p<0.005$; and ${ }^{\star * \star} p<0.0005$. Barplots represents mean \pm SEM of experiments performed in triplicate. $n s$, not significant.

shown to be an indicator of the drug's efficacy (Kotwal et al., 2020), suggesting functional PD-L1 expression in native thyroid glands. The expression of PD-L1 in thyroid cancers has been extensively studied using IHC (Aghajani et al., 2018). PD-L1 positivity is correlated with lower overall survival in anaplastic thyroid cancer (Chintakuntlawar et al., 2017) and worse clinicopathologic characteristics in PTC including reduced progression-free survival, increased lymphovascular invasion, tumor size, and TNM stage (Chowdhury et al., 2016; Shi et al., 2017; Gillanders and O'Neill, 2018). While the prevalence and severity of thyroid cancer differs between males and females, PD-L1 IHC in PTC exhibits no sexbased changes in the percentage of positively staining cells (Aghajani et al., 2018). The bulk RNA-sequencing performed in this study aggregates $\mathrm{PD}-\mathrm{L} 1$ expression in the entire tissue while IHC indicates a proportion of cells that express PD-L1 without providing the degree of expression for the individual cell. Furthermore, our data indicates that $A R$ is significantly downregulated in both male and female thyroid tumors compared to normal tissues. This may partially explain why PD-L1 IHC shows no sex-related differences as androgenmediated PD-L1 attenuation is blunted by the downregulation of AR in male tumors.

To our knowledge, this is the first study identifying a relationship between $\mathrm{AR}$ activity and $\mathrm{PD}-\mathrm{L} 1$ expression in thyroid cancer. Recently, Jiang et al. (2020) characterized AR suppression of PD-L1 in hepatocellular carcinoma. In combination, these findings point to a previously unappreciated role $\mathrm{AR}$ has on $\mathrm{PD}-\mathrm{L} 1$ expression in multiple tissue types and, given that the PD-1/PD-L1 axis is an actionable immunotherapeutic target for various cancers, indicate that AR activity can impact the response to immunotherapy. An ongoing phase II clinical trial is investigating the efficacy of atezolizumab, a humanized PD-L1 monoclonal antibody, in combination with small molecule inhibitors or cytotoxic agents in the most aggressive forms of thyroid cancer (NCT03181100). Preliminary results for this trial were recently reported at the 2020 ASCO Annual Meeting and showed that compared to the historical ATC OS median of 5 months, patients in all three atezolizumab cohorts had a median OS of over 18 months. 


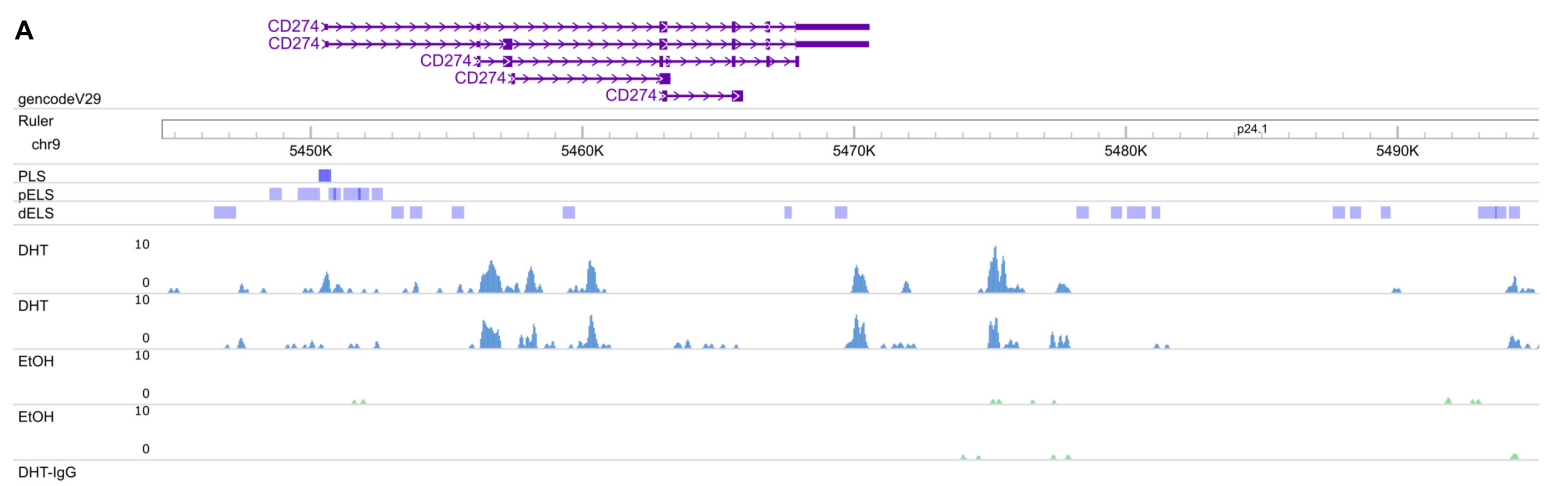

B

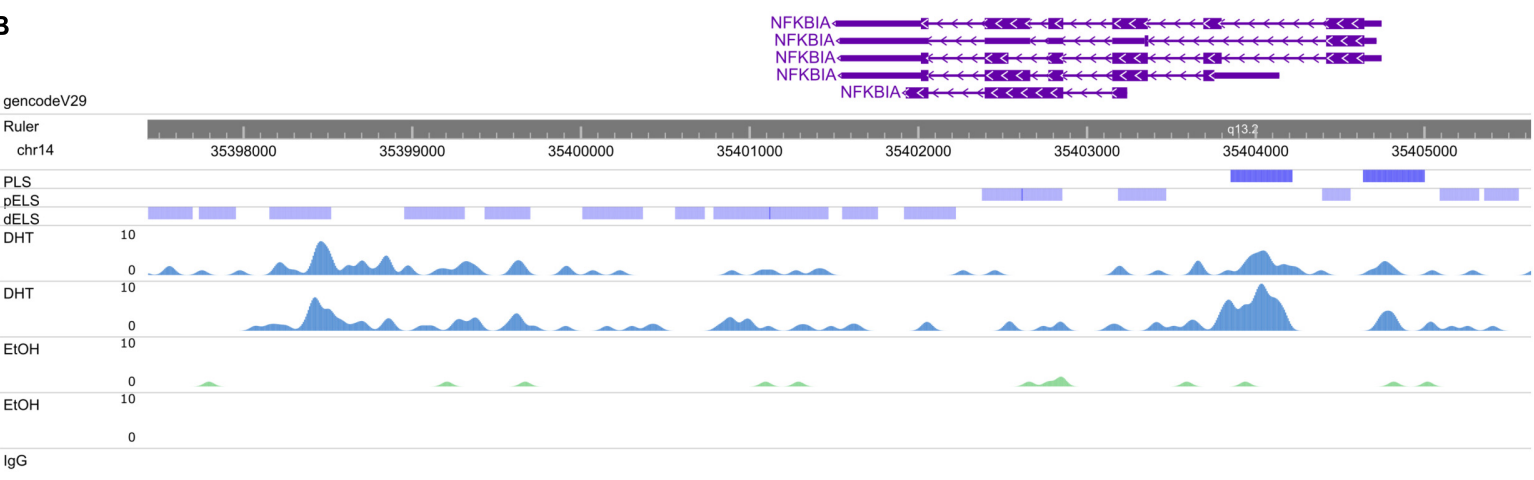

c $8505 \mathrm{c} \quad 84 \mathrm{e} 7$
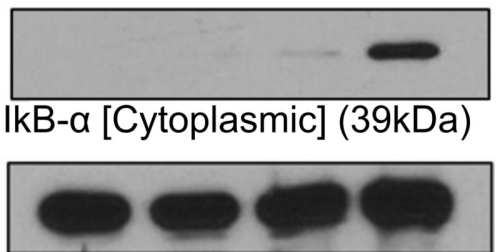

NFkB [Cytoplasmic] (65kDa)
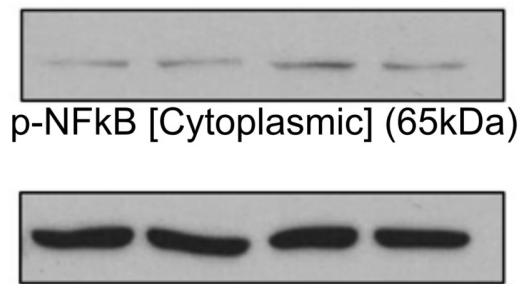

$\beta$-Tubulin [Cytoplasmic] (51kDa)
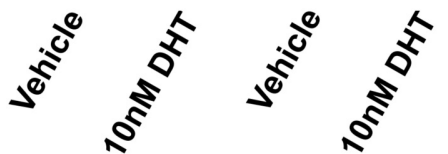

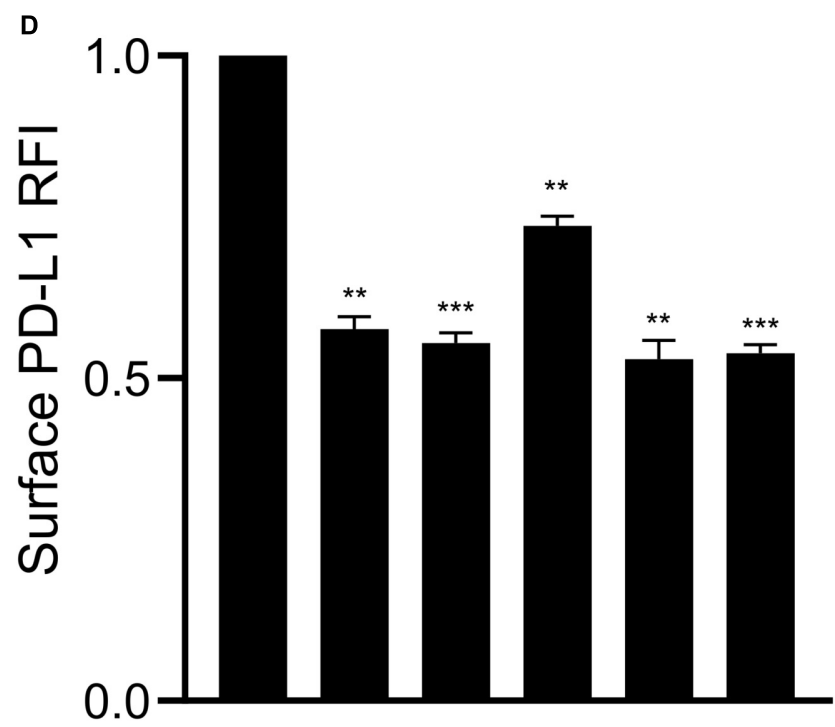

\section{$\mathrm{DHT}$ \\ BMS}

Veh 1nM 10nM Veh 1nM 10nM

FIGURE 3 | Profiling AR binding genome-wide in 8505C-AR cells. CUT\&Tag AR signal at the CD274 gene body (A) and the NFKBIA gene promoter (B). (C) Western blot analysis of cell cytoplasmic fraction from $72 \mathrm{~h}$ vehicle and $10 \mathrm{nM}$ DHT-treated 8505C and 84E7. (D) Surface PD-L1 expression in 84E7 cells co-treated with $10 \mathrm{nM}$ DHT for $48 \mathrm{~h}$ and the IKK inhibitor BMS-345541 (BMS) at $10 \mu \mathrm{M}$ for $24 \mathrm{~h}$. PLS, promoter-like signal; pELS, predicted enhancer-like signal; IgG, isotype control IgG CUT\&Tag. Student $t$-tests paired to control conditions ${ }^{* *} p<0.005$ and ${ }^{* \star *} p<0.0005$. 
Overall, 10 of 38 patients had complete tumor resection after therapy and seven of those patients were alive at the time of the report (Cabanillas et al., 2020).

Similar to Jiang et al. (2020), we identified a direct interaction between AR and the CD274 gene body using CUT\&Tag. However, since we were able to profile AR binding sites genome-wide, we also identified an AR binding signal at the NFKBIA promoter. AR activation may impact NF-kB signaling by increasing IkB $\alpha$ which thereby prevents NF-kB translocation into the nucleus. A decrease in nuclear NF-kB results in a direct reduction in PD-L1 expression due to the complex's involvement in key enhancer-promoter interactions (Chen et al., 2018). However, inhibition of NF-kB signaling using BMS345541 did not reduce PD-L1 expression to the same level as DHT, suggesting AR likely represses PD-L1 via multiple mechanisms. This may include direct inhibition of PD-L1 promoter and enhancer elements as well as modulation of signaling pathways responsible for regulating PD-L1 expression, such as the IFN $\gamma$ pathway.

There is a well-established gender disparity in thyroid cancer incidence. Underlying inflammatory processes in healthy females may predispose them toward developing thyroid cancer (Manole et al., 2001; Tafani et al., 2014). Klein and Flanagan (2016) have stated that sex is a biological variable that should be considered in immunological studies. We postulate that reduced levels of PD-L1 in males allows for continuous immunosurveillance and elimination of nascent tumors, whereas higher levels of PD-L1 in females results in immuno-evasive phenotypes (Mould et al., 2017). In addition to the role of AR in modulating the PD-L1 expression, $\mathrm{ER} \alpha$ activation by $17 \beta$-Estradiol upregulates PD-L1 protein expression, and perhaps contributing to the immune inhibitory environment in women (Yang et al., 2017). Given the gender difference in inflammatory thyroiditis, which can contribute to elevated PD-L1 levels due to expression of cytokines such as IFN $\gamma$, constitutively active immunological processes, lower AR levels and/or estrogen receptor activation in females may lead to elevated PD-L1 levels, thereby contributing to the thyroid cancer gender disparity. However, the present study is limited to only providing correlative data in primary human thyroid carcinomas to suggest the relationship between AR activity and PD-L1. Furthermore, our cell culture model is limited by the use of exogenous expression of AR in an immortalized thyroid cancer cell line which may not represent the physiologic role of AR signaling in vivo. While the $8505 \mathrm{C}$ cell line expresses relatively high levels of AR compared to other thyroid cancer cell lines, it is evident from our results that these cells do not respond to DHT stimulation. Further investigation into the role of AR in primary thyroid cancer cells, particularly PTC cells, is warranted to establish a potential underlying mechanism and its role in the disparity in incidence.

In summary, this study suggests that PD-L1 is downregulated by androgens in thyroid cancer cells in an AR-dependent mechanism. This putative mechanism is corroborated by the inverse correlation of AR and PD-L1 expression in primary human tissues and the higher frequency of PD-L1 expression in female PTC tissues. Genome-wide profiling of AR binding sites reveals both direct activity at the PD-L1 locus as well as indirect regulatory mechanisms via inhibition of NF$\mathrm{kB}$ through upregulation of $\mathrm{IkB} \alpha$. These findings provide a foundation for investigating the role PD-L1 expression has in the pathogenesis of thyroid cancer and the disparity in incidence between the genders.

\section{DATA AVAILABILITY STATEMENT}

The datasets presented in this study can be found in online repositories. The names of the repository/repositories and accession number(s) can be found below: https://www.ncbi.nlm. nih.gov/bioproject/?term=PRJNA698545, PRJNA698545.

\section{ETHICS STATEMENT}

The studies involving human participants were reviewed and approved by the Institutional Review Board at New York Medical College, New York Eye, and Ear Infirmary. The patients/participants provided their written informed consent to participate in this study.

\section{AUTHOR CONTRIBUTIONS}

TO'C, RKT, and JG conceived of the study and designed the experiments. TO'C, SD, AG, and MJ performed the experiments. TO'C, SD, and TR analyzed and interpreted the data. ES, AM, CI, and $\mathrm{HI}$ provided clinical expertise and primary tissue. TO'C and $\mathrm{SD}$ wrote the manuscript. All authors contributed to the article and approved the submitted version.

\section{FUNDING}

This work was supported by the New York State Empire Clinical Research Investigator Program (RKT awardee), Department of Otolaryngology at New York Medical College, and the 2019 AHNS Endocrine Surgery Section Eisai Research Award (SD awardee).

\section{ACKNOWLEDGMENTS}

We would like to thank Zbigniew Darzynkiewicz, Jiangwei Li, and Dorota Halicka for their assistance with flow cytometry experiments and Anne Solas of the New York Medical College Histopathology core for assistance with IHC.

\section{SUPPLEMENTARY MATERIAL}

The Supplementary Material for this article can be found online at: https://www.frontiersin.org/articles/10.3389/fcell.2021. 663130/full\#supplementary-material

Supplementary Table 1 | Primary antibodies used for western blotting. 


\section{REFERENCES}

Aghajani, M. J., Yang, T., McCafferty, C. E., Graham, S., Wu, X., and Niles, N. (2018). Predictive relevance of programmed cell death protein 1 and tumorinfiltrating lymphocyte expression in papillary thyroid cancer. Surgery 163, 130-136. doi: 10.1016/j.surg.2017.04.033

Ahn, S., Kim, T. H., Kim, S. W., Ki, C. S., Jang, H. W., Kim, J. S., et al. (2017). Comprehensive screening for PD-L1 expression in thyroid cancer. Endocr. Relat. Cancer 24, 97-106. doi: 10.1530/erc-16-0421

Álvarez-Sierra, D., Marín-Sánchez, A., Ruiz-Blázquez, P., de Jesús Gil, C., IglesiasFelip, C., González, Ó, et al. (2019). Analysis of the PD-1/PD-L1 axis in human autoimmune thyroid disease: Insights into pathogenesis and clues to immunotherapy associated thyroid autoimmunity. J. Autoimmun. 103:102285. doi: 10.1016/j.jaut.2019.05.013

Ansar Ahmed, S., Dauphinee, M. J., and Talal, N. (1985). Effects of short-term administration of sex hormones on normal and autoimmune mice. J. Immunol. 134, 204-210.

Broad Institute (2019). Picard Toolkit. Github. Available online at: https://github. com/broadinstitute/picard (accessed January 1, 2021).

Burke, J. R., Pattoli, M. A., Gregor, K. R., Brassil, P. J., MacMaster, J. F., McIntyre, K. W., et al. (2003). BMS-345541 is a highly selective inhibitor of IкB kinase that binds at an allosteric site of the enzyme and blocks NF- $\kappa \mathrm{B}$-dependent transcription in mice*. J. Biol. Chem. 278, 1450-1456. doi: 10.1074/jbc. m209677200

Cabanillas, M. E., Dadu, R., Ferrarotto, R., Liu, S., Fellman, B. M., Gross, N. D., et al. (2020). American society of clinical oncology. Virt. Annu. Meet. 2020:6514.

Cantara, S., Bertelli, E., Occhini, R., Regoli, M., Brilli, L., Pacini, F., et al. (2019). Blockade of the programmed death ligand 1 (PD-L1) as potential therapy for anaplastic thyroid cancer. Endocrine 64, 122-129. doi: 10.1007/s12020-01901865-5

Chen, H., Li, C., Peng, X., Zhou, Z., Weinstein, J. N., Cancer Genome Atlas Research Network, et al. (2018). A pan-cancer analysis of enhancer expression in nearly 9000 patient samples. Cell 173, 386-399.e12.

Chintakuntlawar, A. V., Rumilla, K. M., Smith, C. Y., Jenkins, S. M., Foote, R. L., Kasperbauer, J. L., et al. (2017). Expression of PD-1 and PD-L1 in anaplastic thyroid cancer patients treated with multimodal therapy: results from a retrospective study. J. Clin. Endocrinol. Metab. 102, 1943-1950. doi: 10.1210/jc.2016-3756

Chowdhury, S., Veyhl, J., Jessa, F., Polyakova, O., Alenzi, A., MacMillan, C., et al. (2016). Programmed death-ligand 1 overexpression is a prognostic marker for aggressive papillary thyroid cancer and its variants. Oncotarget 7, 32318-32328. doi: 10.18632 /oncotarget.8698

D’Andréa, G., Lassalle, S., Guevara, N., Mograbi, B., and Hofman, P. (2021). From biomarkers to therapeutic targets: the promise of PD-L1 in thyroid autoimmunity and cancer. Theranostics 11, 1310-1325. doi: 10.7150/thno. 50333

Dobin, A., Davis, C. A., Schlesinger, F., Drenkow, J., Zaleski, C., Jha, S., et al. (2013). STAR: ultrafast universal RNA-seq aligner. Bioinformatics 29, 15-21. doi: 10.1093/bioinformatics/bts635

ENCODE Project Consortium, Moore, J. E., Purcaro, M. J., Pratt, H. E., Epstein, C. B., Shoresh, N., et al. (2020). Expanded encyclopaedias of DNA elements in the human and mouse genomes. Nature 583, 699-710.

Gillanders, S. L., and O'Neill, J. P. (2018). Prognostic markers in well differentiated papillary and follicular thyroid cancer (WDTC). Eur. J. Surg. Oncol. 44, 286296. doi: 10.1016/j.ejso.2017.07.013

Goldman, M., Craft, B., Brooks, A., Zhu, J., and Haussler, D. (2018). The UCSC Xena Platform for cancer genomics data visualization and interpretation. BioRxiv [Preprint]. Available online at: https://www.biorxiv.org/content/10. 1101/326470v1.abstract (accessed June 2019).

Goldspiel, B. R., and Kohler, D. R. (1990). Flutamide: an antiandrogen for advanced prostate cancer. DICP 24, 616-623. doi: 10.1177/106002809002400612

Jiang, G., Shi, L., Zheng, X., Zhang, X., Wu, K., Liu, B., et al. (2020). Androgen receptor affects the response to immune checkpoint therapy by suppressing PDL1 in hepatocellular carcinoma. Aging 12, 11466-11484. doi: 10.18632/aging. 103231

Jones, M., O’Connell, T., Zhao, H., Darynkiewicz, Z., Gupta, A., Buchsbaum, J., et al. (2021). Androgen receptor activation decreases proliferation in thyroid cancer cells. J. Cell. Biochem. [Epub ahead of print]. doi: 10.1002/jcb.29934
Kaya-Okur, H. S., Wu, S. J., Codomo, C. A., Pledger, E. S., Bryson, T. D., Henikoff, J. G., et al. (2019). CUT\&Tag for efficient epigenomic profiling of small samples and single cells. Nat. Commun. 10:1930.

Kilfoy, B. A., Zheng, T., Holford, T. R., Han, X., Ward, M. H., Sjodin, A., et al. (2009). International patterns and trends in thyroid cancer incidence, 1973-2002. Cancer Caus. Control 20, 525-531. doi: 10.1007/s10552-008-9260-4

Klein, S. L., and Flanagan, K. L. (2016). Sex differences in immune responses. Nat. Rev. Immunol. 16, 626-638. doi: 10.1038/nri.2016.90

Kotwal, A., Kottschade, L., and Ryder, M. (2020). PD-L1 inhibitor-induced thyroiditis is associated with better overall survival in cancer patients. Thyroid 30, 177-184. doi: 10.1089/thy.2019.0250

Lawrence, M. S., Stojanov, P., Polak, P., Kryukov, G. V., Cibulskis, K., Sivachenko, A., et al. (2013). Mutational heterogeneity in cancer and the search for new cancer-associated genes. Nature 499, 214-218.

Li, B., and Dewey, C. N. (2011). RSEM: accurate transcript quantification from RNA-Seq data with or without a reference genome. BMC Bioinform. $12: 323$.

Li, H. (2013). Aligning sequence reads, clone sequences and assembly contigs with BWA-MEM. arXiv [Preprint]. Available online at: http://arxiv.org/abs/1303. 3997 (accessed June 2019).

Li, H., Handsaker, B., Wysoker, A., Fennell, T., Ruan, J., Homer, N., et al. (2009). The sequence alignment/map format and SAM tools. Bioinformatics 25, 20782079.

Magri, F., Capelli, V., Rotondi, M., Leporati, P., La Manna, L., Ruggiero, R., et al. (2012). Expression of estrogen and androgen receptors in differentiated thyroid cancer: an additional criterion to assess the patient's risk. Endocr. Relat. Cancer 19, 463-471. doi: 10.1530/erc-11-0389

Manole, D., Schildknecht, B., Gosnell, B., Adams, E., and Derwahl, M. (2001). Estrogen promotes growth of human thyroid tumor cells by different molecular mechanisms. J. Clin. Endocrinol. Metab. 86, 1072-1077. doi: 10.1210/jc.86.3. 1072

Martin, M. (2011). Cutadapt removes adapter sequences from high-throughput sequencing reads. EMBnet. J. 17, 10-12. doi: 10.14806/ej.17.1.200

Morris, L. G. T., Tuttle, R. M., and Davies, L. (2016). Changing trends in the incidence of thyroid cancer in the United States. JAMA Otolaryngol Head Neck Surg. 142, 709-711. doi: 10.1001/jamaoto.2016.0230

Mould, R. C., van Vloten, J. P., AuYeung, A. W. K., Karimi, K., and Bridle, B. W. (2017). Immune responses in the thyroid cancer microenvironment: making immunotherapy a possible mission. Endocr. Relat. Cancer 24, T311-T329.

Naugler, W. E., Sakurai, T., Kim, S., Maeda, S., Kim, K., Elsharkawy, A. M., et al. (2007). Gender disparity in liver cancer due to sex differences in MyD88dependent IL-6 production. Science 317, 121-124. doi: 10.1126/science. 1140485

Ngo, S. T., Steyn, F. J., and McCombe, P. A. (2014). Gender differences in autoimmune disease. Front. Neuroendocrinol. 35:347-369. doi: 10.1016/j.yfrne. 2014.04.004

Nguyen, Q. T., Lee, E. J., Huang, M. G., Park, Y. I., Khullar, A., and Plodkowski, R. A. (2015). Diagnosis and treatment of patients with thyroid cancer. Am Health Drug Benefits 8, 30-40.

Nosrati, A., Tsai, K. K., Goldinger, S. M., Tumeh, P., Grimes, B., Loo, K., et al. (2017). Evaluation of clinicopathological factors in PD-1 response: derivation and validation of a prediction scale for response to PD-1 monotherapy. Br. J. Cancer 116, 1141-1147. doi: 10.1038/bjc.2017.70

Özdemir, B. C., and Dotto, G.-P. (2019). Sex hormones and anticancer immunity. Clin. Cancer Res. 25, 4603-4610. doi: 10.1158/1078-0432.ccr-19-0137

Rahbari, R., Zhang, L., and Kebebew, E. (2010). Thyroid cancer gender disparity. Fut. Oncol. 6, 1771-1779. doi: 10.2217/fon.10.127

Rajoria, S., Suriano, R., Shanmugam, A., Wilson, Y. L., Schantz, S. P., Geliebter, J., et al. (2010). Metastatic phenotype is regulated by estrogen in thyroid cells. Thyroid 20, 33-41. doi: 10.1089/thy.2009.0296

Ramírez, F., Dündar, F., Diehl, S., Grüning, B. A., and Manke, T. (2014). deepTools: a flexible platform for exploring deep-sequencing data. Nucleic Acids Res. 42, W187-W191.

Ritprajak, P., and Azuma, M. (2015). Intrinsic and extrinsic control of expression of the immunoregulatory molecule PD-L1 in epithelial cells and squamous cell carcinoma. Oral Oncol. 51, 221-228. doi: 10.1016/j.oraloncology.2014. 11.014 
Ryu, A. H., Eckalbar, W. L., Kreimer, A., Yosef, N., and Ahituv, N. (2017). Use antibiotics in cell culture with caution: genome-wide identification of antibiotic-induced changes in gene expression and regulation. Sci. Rep. 7:7533.

Salameh, W. A., Redor-Goldman, M. M., Clarke, N. J., Reitz, R. E., and Caulfield, M. P. (2010). Validation of a total testosterone assay using high-turbulence liquid chromatography tandem mass spectrometry: total and free testosterone reference ranges. Steroids 75, 169-175. doi: 10.1016/j.steroids.2009.11.004

Shi, R.-L., Qu, N., Luo, T.-X., Xiang, J., Liao, T., Sun, G.-H., et al. (2017). Programmed death-ligand 1 expression in papillary thyroid cancer and its correlation with clinicopathologic factors and recurrence. Thyroid 27, 537-545. doi: 10.1089/thy.2016.0228

Siegel, R. L., Miller, K. D., and Jemal, A. (2020). Cancer statistics, 2020. CA Cancer J. Clin. 70, 7-30.

Song, Y. S., Won, J.-K., Yoo, S.-K., Jung, K. C., Kim, M. J., Kim, S.-J., et al. (2018). Comprehensive transcriptomic and genomic profiling of subtypes of follicular variant of papillary thyroid carcinoma. Thyroid 28, 1468-1478. doi: 10.1089/thy.2018.0198

Su, X., Li, Z., He, C., Chen, W., Fu, X., and Yang, A. (2016). Radiation exposure, young age, and female gender are associated with high prevalence of RET/PTC1 and RET/PTC3 in papillary thyroid cancer: a meta-analysis. Oncotarget 7 , 16716-16730. doi: 10.18632/oncotarget.7574

Tafani, M., De Santis, E., Coppola, L., Perrone, G. A., Carnevale, I., Russo, A., et al. (2014). Bridging hypoxia, inflammation and estrogen receptors in thyroid cancer progression. Biomed. Pharmacother. 68, 1-5. doi: 10.1016/j.biopha.2013. 10.013

Thorsson, V., Gibbs, D. L., Brown, S. D., Wolf, D., Bortone, D. S., Ou Yang, T.-H., et al. (2019). The immune landscape of cancer. Immunity 51, 411-412.
Wilms, T., Gu, X., Boldrup, L., Coates, P. J., Fahraeus, R., Wang, L., et al. (2020). PD-L1 in squamous cell carcinoma of the oral tongue shows gender-specific association with prognosis. Oral Dis. 26, 1414-1423. doi: 10.1111/odi.13414

Yang, L., Huang, F., Mei, J., Wang, X., Zhang, Q., Wang, H., et al. (2017). Posttranscriptional control of PD-L1 expression by $17 \beta$-estradiol via PI3K/Akt signaling pathway in ER $\alpha$-positive cancer cell lines. Int. J. Gynecol. Cancer 27, 196-205. doi: 10.1097/igc.0000000000000875

Yorke, E., Melck, A., and Wiseman, S. M. (2016). Impact of sex on the clinicopathological characteristics and prognosis of papillary thyroid cancer. Can. J. Surg. 59, 287-288. doi: 10.1503/cjs.003816

Conflict of Interest: The authors declare that the research was conducted in the absence of any commercial or financial relationships that could be construed as a potential conflict of interest.

Publisher's Note: All claims expressed in this article are solely those of the authors and do not necessarily represent those of their affiliated organizations, or those of the publisher, the editors and the reviewers. Any product that may be evaluated in this article, or claim that may be made by its manufacturer, is not guaranteed or endorsed by the publisher.

Copyright (c) 2021 O’Connell, Dadafarin, Jones, Rodríguez, Gupta, Shin, Moscatello, Iacob, Islam, Tiwari and Geliebter. This is an open-access article distributed under the terms of the Creative Commons Attribution License (CC BY). The use, distribution or reproduction in other forums is permitted, provided the original author(s) and the copyright owner(s) are credited and that the original publication in this journal is cited, in accordance with accepted academic practice. No use, distribution or reproduction is permitted which does not comply with these terms. 